\title{
Simulation study of air-induced segregation of equal-sized bronze and glass particles
}

\author{
C. Zeilstra, M. A. van der Hoef, and J. A. M. Kuipers \\ Department of Science \& Technology, University of Twente, P.O. Box 217, 7500 AE Enschede, The Netherlands
}

(Received 18 November 2005; revised manuscript received 24 March 2006; published 24 July 2006; publisher error corrected 27 July 2006 )

\begin{abstract}
We have investigated the effect of air on the density segregation of fine, equal-sized bronze and glass particles under vertical sinusoidal vibrations by numerical simulation, using a hybrid granular dynamicscomputational fluid dynamics model. We find both the bronze-on-top and the sandwich configurations as observed in previous experiments. The simulations allow us to give a detailed explanation of the phenomena. The key observation is the precise timing of the decompaction of the bed with the oscillating gas flow, which will cause the bronze to move to the top, due to the difference in acceleration from the air drag. For higher frequencies, the different inertia in collisions will cause the bronze clusters on top to sink through the bed, leading to a sandwich formation.
\end{abstract}

DOI: 10.1103/PhysRevE.74.010302

PACS number(s): 45.70.Mg, 02.70.Ns, 02.70.Bf

Granular media subject to vertical vibrations have been the subject of much research in the past decades, not only out of intrinsic scientific interest, but also because of the numerous applications in industry [1]. Most of these studies were concerned with relatively large particles $(>1 \mathrm{~mm})$, where the effects of air can be safely ignored. However, for smaller particles, the effects of air cannot be neglected and a whole new range of interesting phenomena may occur. Pak et al. [2] found that the surrounding air plays a crucial role in heap generation, whereas Möbius et al. [3] found that the air pressure has a profound effect on the rising time of a large intruder, submerged in a bed of smaller particles. However, one of the most remarkable observations was made by Burtally et al. $[4,5]$, who found experimentally that a mixture of fine bronze and glass particles of equal size (55 $\mu \mathrm{m}$ radius) segregate under vertical vibrations, but only when air is forced through the granular bed. The effect appears to be very robust, and was also found in numerical simulations with very few particles (3000) and a simplified description of the gas phase [6], yet it is currently not understood in detail why this phenomenon happens. The goal of this work is to shed light on the mechanism underlying these remarkable effects, by means of extensive numerical simulations of systems containing 30000 spherical particles.

In the experiments of Burtally et al., it was found that the characteristics of the density segregation were directly dependent on the shaking amplitude and frequency. They summarized their findings in a "phase diagram" of acceleration vs frequency in which each area corresponds to a typical segregation behavior. During vibration, two major forms of segregation can be observed. The first shows clustering of the bronze particles on top of the granular bed ("bronze-ontop"), the second results in clustering of the bronze particles between two glass layers ("sandwich"). Additionally, some metastable regions have been found that show fluctuations between bronze-on-top and sandwich formations. It is clear that the air plays a dominant role in these effects, since no significant segregation is observed under vacuum conditions. One of the effects of the air is that particles are slowed down due to the gas drag. This effect will be stronger for the glass particles due to their lower inertia, however, this would not explain why the heavy particles cluster at the top. Moreover, it was found both experimentally [5] and numerically [6] that air must be forced through the granular bed for significant segregation to occur, implying that the mere presence of air is not sufficient.

In order to get a better insight into the mechanisms responsible for these segregation phenomena, we have performed computer simulations of the glass-bronze bed under vertical sinusoidal vibration, using a hybrid granular dynamics-computational fluid dynamics code [7]. For the dynamics of the granular particles, a three-dimensional linear spring and dashpot type soft sphere collision model is used, including tangential friction. In all the simulations, the coefficient of restitution, which determines the amount of kinetic energy that is dissipated in a collision, is set to the values of 0.97 for the normal direction and 0.33 for the tangential direction, which were found to be the most realistic values in a previous simulation study [8]. For the particle-wall interaction we use the same collision parameters as for the particleparticle interaction. The friction coefficient is set to 0.1 . For the gas phase, we divide the domain through which the box moves into computational cells and evaluate the volumeaveraged Navier-Stokes equations for each cell to obtain the pressure and the velocity field. The shear viscosity $\eta$, average pressure $P$, and density $\rho_{g}$ of the gas phase are set to match the properties of air at ambient conditions: $\eta=1.8$ $\times 10^{-5} \mathrm{~kg} / \mathrm{ms}, P=1 \mathrm{bar}$, and $\rho_{g}=1.2 \mathrm{~kg} / \mathrm{m}^{3}$. For these settings, viscous shear layers are not expected to play a role. The bulk viscosity is set to zero. Since our box is of limited depth, we evaluate the gas velocities in only two dimensions. There is a full two-way coupling between the granular particles and the gas phase, where the effective momentum exchange is calculated from the Ergun and Wen and Yu drag force correlations [9]. For a comprehensive overview of the model, see [7].

The system that we studied in the simulations contains 7500 bronze $\left(\rho=8900 \mathrm{~kg} / \mathrm{m}^{3}\right)$ mixed with 22500 glass $\left(\rho=2525 \mathrm{~kg} / \mathrm{m}^{3}\right)$ particles of equal size (55 $\mu \mathrm{m}$ radius). A Gaussian size distribution was applied $(\sigma=7.5 \mu \mathrm{m})$, to avoid excessive ordering of the bed under less vigorous shaking conditions. The box is a closed system with a ground area of $8.25 \times 0.65 \mathrm{~mm}$, and the granular bed height is approximately $6.5 \mathrm{~mm}$. We have studied different sets of vibration 


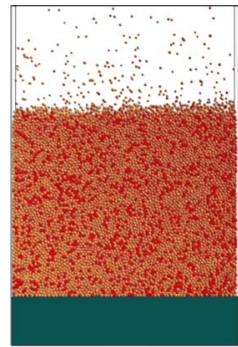

$\mathrm{t}=0.0 \mathrm{~s}$

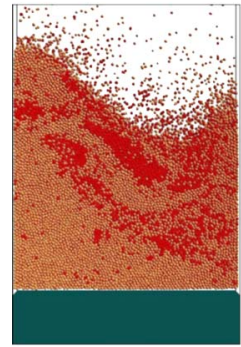

$t=0.6 s$

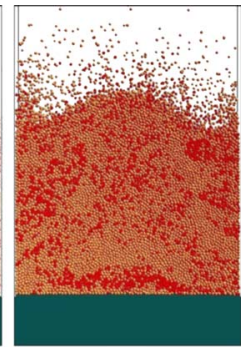

$\mathrm{t}=0.2 \mathrm{~s}$

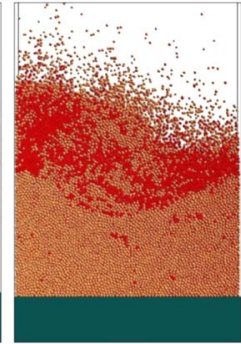

$\mathrm{t}=0.9 \mathrm{~s}$
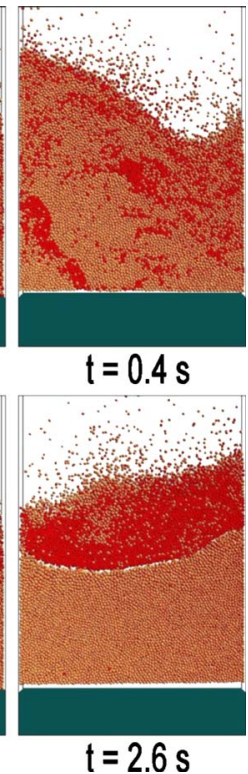

FIG. 1. (Color online) A series of snapshots leading to the bronze-on-top system $(A=0.9 \mathrm{~mm}, f=55 \mathrm{~Hz}, \Gamma=11)$. Small bronze clusters are formed which migrate to the surface of the granular bed where a layer of bronze is formed that eventually covers the entire width of the box. After segregation, the upper bronze particles are quite active and the bed surface shows severe tilting, however, bronze remains on top.

parameters corresponding to the various regions in the experimental phase diagram, and found that the model is able to successfully predict the observations by Burtally et al., although the transient behavior of the metastable regions could not be reproduced. We also found that no significant segregation occurred under vacuum conditions, or when there is no forcing of air through the system by having an open top and a porous bottom plate, in accordance with the experimental findings. It is interesting to note that in a similar simulation study with 3000 particles by Milburn et al. [10], fluid-driven convection rolls were observed within the separate layers, which strengthened the separation mechanism. In our simulations we did not observe any significant fluid-driven convection, which is probably due to the larger bedheight which prevents Faraday tilting.

We next focus on a bronze-on-top system driven with a vibration frequency $f$ of $55 \mathrm{~Hz}$ and a vibration amplitude $A$ in the vertical direction of $0.9 \mathrm{~mm}$, leading to a dimensionless acceleration $\Gamma=A(2 \pi f)^{2} / g$ of 11 . Initially, the system is well mixed, however as time proceeds, the bronze and glass start to segregate, see Fig. 1. For all of our simulations at atmospheric pressure, we found that the granular bed moves in phase with the bottom plate. Animations reveal that within a single vibration cycle during segregation (i.e., around $t$ $=0.3 \mathrm{~s}$ ), initially bronze and glass are going up together with the bottom plate. When the bed is lifted from the plate, the glass particles loose momentum more quickly than the heavy bronze particles, and one can clearly observe the glass particles flowing downwards relative to the bronze particles. However, when the bed is falling down on the bottom plate, the opposite phenomenon is observed. Again the bronze retains its momentum (but now downwards) longer than the

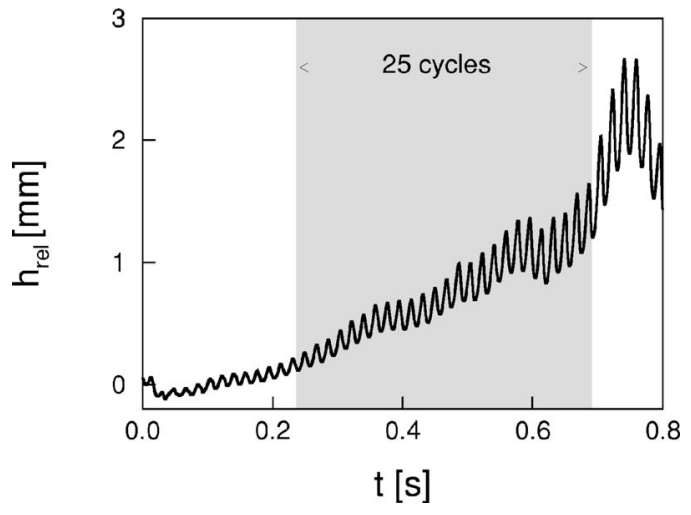

FIG. 2. Position of the bronze relative to the glass as a function of time for the bronze-on-top system. For $h_{\text {rel }}=0$, the system is completely mixed, whereas $h_{\text {rel }} \sim 3$ corresponds to the situation as shown in Fig. 1 on the bottom right. The shaded area marks the data that we used for further analysis by averaging, as shown in Fig. 3.

glass, however the relative movement is much less in this case since the bed is compactified, in particular in the bottom region. The observation that compaction can lock particles against further movement has been reported previously for the Faraday heap [11] and for the Brazil nut effect [12,13].

Next, we want to quantify the visual observations. In Fig. 2 we show the relative position of the bronze and glass particles $h_{\text {rel }}=\left\langle h_{\text {bronze }}\right\rangle-\left\langle h_{\text {glass }}\right\rangle$, where $\left\langle h_{i}\right\rangle$ is the average height of particle species $i$. It can be seen that the bronze gradually moves upward. Since the motion of the granular bed is in phase with the bottom plate, we average the data of interest over 25 vibration cycles in order to minimize the effect of the fluctuations, where the averaging window is indicated in Fig. 2. We found that for this particular time frame the bronze-to-top movement was most clearly observed, and less pronounced for times $<0.2$ and $>0.7$. We start the analysis on the basis of Fig. 3 at the point when the bottom plate moves through the equilibrium position. When the granular bed looses contact with the bottom plate, a void is formed between them. As the size of the void increases, the pressure drops so that gas is sucked into the granular bed causing negative gas accelerations on both particle species. As the size of the void starts to decrease, the pressure beneath the granular bed increases and the gas accelerations become positive. The lower section of the granular bed regains contact with the bottom plate and as the granular bed is compacting, the vibration cycle is complete. During this vibration cycle, two regions are distinguished: one of negative and one of positive gas accelerations. These accelerations are a direct result of the interaction between the movements of the granular bed and the bottom plate, which has the effect of a piston, inducing positive and negative pressure gradients within a cycle. The forces acting on the glass and bronze particles are equal (since the particles have equal size), however the accelerations are different, due to the different mass. Note that these accelerations can be almost an order of magnitude larger than gravity. It can be seen from Fig. 3 that when the system is most densely packed, $h_{\text {rel }}$ does not change significantly, i.e., there is no relative movement. Only when the packing fraction decreases, that is, when the granu- 


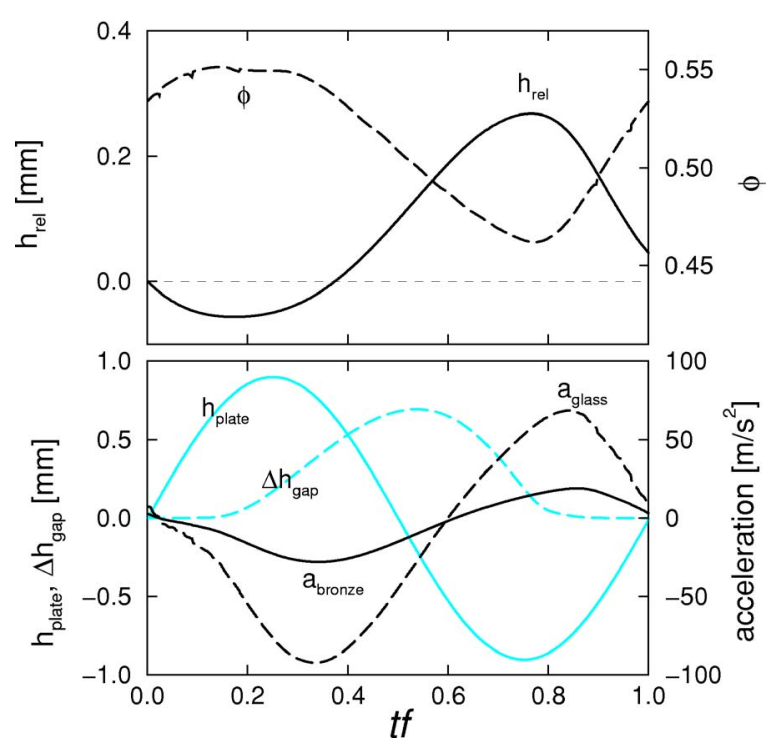

FIG. 3. (Color online) Evolution of the quantities of interest within one cycle for the bronze-on-top system, obtained by averaging over 25 cycles (see Fig. 2). Top graph: relative position $h_{\text {rel }}$ of the bronze and glass particles (black line) and packing fraction $\phi$ of the bed (black dashed line). Lower graph: position $h_{\text {plate }}$ of the bottom plate (gray line) and the width $\Delta h_{\text {gap }}$ of the gap between the granular bed and bottom plate (gray dashed line). The black solid and black dashed line represent the average acceleration in the vertical direction due to the total force from the gas phase on a bronze and a glass particle, respectively. Note that all quantities are periodic in time, except for the relative position of the bronze and glass particles (black line in top graph), which ends up slightly higher at $t f=1$ (as compared to $t f=0$ ) due to the bed compaction starting at $t f=0.8$, which hinders the relative movement of the particles. Because of this effect, bronze will gradually move to the top.

lar bed expands in free flight, a significant relative movement occurs. In the regime of negative accelerations, glass moves downward relative to the bronze. At $t f=0.6$, the direction of the accelerations is changed, causing the relative movement of glass to slow down, come to a stop, and eventually reverse: glass is going up (i.e., $h_{\text {rel }}$ becomes smaller again). However, at this time the packing fraction starts to increase since contact between granular bed and bottom plate has been regained: compaction starts from the bottom plate up. Due to the compaction the relative movement becomes much more difficult, so that the bronze cannot regain the position it had at the beginning of the cycle, but ends up slightly higher. After a number of such cycles, the bronze will have moved to the top of the granular bed (see Fig. 2). Note that the difference in gravitational force on the bronze and glass particles does not play a role in this mechanism, but rather the difference in the particle's inertia.

We next focus on a "sandwich" system, which is obtained by setting the vibration parameters to $f=130 \mathrm{~Hz}$ and $A=0.147 \mathrm{~mm}(\Gamma=10)$. In Fig. 4 we show some snapshots of the simulation. Note that the time scale on which the layer is formed is much longer than for the bronze-on-top system, which already suggests that another mechanism will be responsible for the observed behavior. We find that initially the bronze moves to the top, although at a rate which is a factor

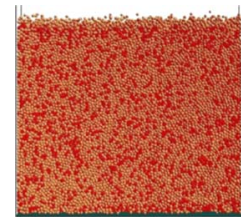

$t=0.0 s$

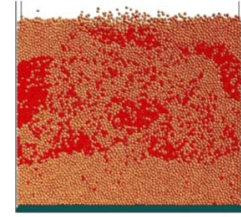

$t=5.0 s$

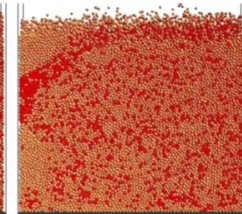

$t=2.0 \mathrm{~s}$

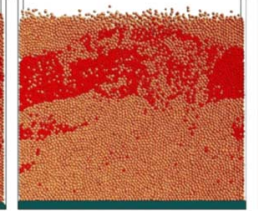

$\mathrm{t}=9.0 \mathrm{~s}$

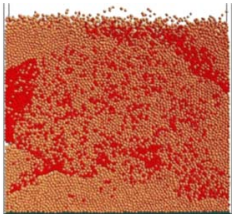

$t=4.0 s$

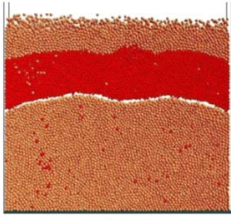

$\mathrm{t}=21.0 \mathrm{~s}$
FIG. 4. (Color online) A series of snapshots for the sandwich system $(A=0.147 \mathrm{~mm}, f=130 \mathrm{~Hz}, \Gamma=10)$. After the formation of the glass-bronze-glass sandwich, the configuration was stable for the time that we simulated $(60 \mathrm{~s})$.

of 25 lower compared to the previous case. How can this difference be explained? Due to the smaller amplitude, the void between granular bed and bottom plate is much smaller for the sandwich than for the bronze-on-top system (1.5 vs 6.5 particle diameters). The time of flight of the granular layer will be smaller for the sandwich system, resulting in a increased packing fraction, varying between 0.56 and 0.59 . This will make it more difficult for the particles to move relative to each other. However, after some of the bronze has clustered on top, a completely different phenomenon is observed. When a bronze cluster at the top becomes sufficiently large, it becomes too heavy for the bed to support it, and it suddenly "falls" through the bed, setting up a large-scale convection roll. Within this motion, the glass-rich layer just below the bronze cluster is pushed to the bottom where it compactifies, so that the bronze cluster cannot reach the bottom, but ends up in the middle of the bed. This happens first at both side walls, but eventually all top bronze clusters sink through the bed, so that a bronze-rich middle layer is formed, leaving an almost pure glass layer at the top. At the same time, the weak "bronze-to-top" mechanism that is still at play causes the middle bronze-rich layer and the bottom glass-rich layer to exchange their impurities. The final result is an almost perfect separation of glass and bronze into a sandwich structure. The question that arises is which effect is responsible for the sinking of the bronze clusters: (i) the difference in heavy mass, i.e., the different gravity force on the bronze and glass particles, or (ii) the difference in inertial mass in collisions, which will cause a bronze cluster to penetrate the glass layer when pounding on it in the second part of the vibration cycle. To answer this question we have performed a purely artificial simulation, where the particles have the same heavy mass (i.e., they experience that same gravity force), while keeping the inertial mass to the original values of bronze and glass. In that case, we still find the sinking of the bronze clusters, although less pronounced, so that the final result is a mixed sandwich and bronze-on-top state. By contrast, for a system where both species have the same inertial mass in the collisions, while the heavy masses are those of bronze and glass, we find no sinking of bronze clusters. It can thus be concluded that the sinking of the 
bronze cluster is primarily caused by the higher inertial mass of the bronze phase, and that the fact that the bronze is heavier plays a minor role.

In conclusion, we have found that most of the phenomena that have been observed experimentally for vertically shaken beds of glass and bronze particles are captured by our hybrid granular dynamics-computational fluid dynamics model. In particular, we found both the bronze-on-top and the sandwich structure for the same shaking conditions as in the experiments, and also that these structures disappeared when the interaction of the particles with the air was switched off. Only the metastable regions, fluctuating between bronze-ontop and sandwich formations, are not observed. We suspect that this is caused by the limited size of the system $(8.25$ $\times 0.65 \times 6.5 \mathrm{~mm}$ ) that we studied. We found that two distinct mechanisms are responsible for segregation: (i) the differ- ence in acceleration from the gas drag causes the bronze to move to the top, due to the pumping of air in combination with the cyclic compaction and decompaction of the bed, and (ii) the difference in inertial mass in collisions causes the bronze clusters on top to penetrate the glass-rich layer below in the second part of the cycle, when the falling bed is hitting the bottom plate. For lower frequencies and higher amplitudes, the first mechanism dominates due to the longer period of free flight, and bronze will always move to the top. For higher frequencies and lower amplitudes, the combination of mechanism (i) and (ii) leads to the sandwich formation.

We thank D. van der Meer for discussions. This work is part of the research program of the Stichting FOM, which is financially supported by NWO.
[1] H. M. Jaeger, S. R. Nagel, and R. P. Behringer, Rev. Mod. Phys. 68, 1259 (1996).

[2] H. K. Pak, E. Van Doorn, and R. P. Behringer, Phys. Rev. Lett. 74, 4643 (1995).

[3] M. E. Möbius, B. E. Lauderdale, S. R. Nagel, and H. M. Jaeger, Nature (London) 414, 270 (2001).

[4] N. Burtally, P. J. King, and M. R. Swift, Science 295, 1877 (2002).

[5] N. Burtally, P. J. King, M. R. Swift, and M. Leaper, Granular Matter 5, 57 (2003).

[6] P. Biswas, P. Sánchez, M. R. Swift, and P. J. King, Phys. Rev. E 68, 050301(R) (2003).

[7] M. A. van der Hoef, M. Ye, M. van Sint Annaland, A. T. Andrews IV, S. Sundaresan, and J. A. M. Kuipers, Adv. Chem.
Eng. 31, 65 (2006).

[8] M. J. V. Goldschmidt, J. A. M. Kuipers, and W. P. M. van Swaaij, Chem. Eng. Sci. 56, 571 (2001).

[9] D. Gidaspow, Multiphase Flow and Fluidization (Academic Press, Boston, 1994).

[10] R. J. Milburn, M. R. Swift, and P. J. King, in Powders and Grains, edited by R. Garcia-Rojo, H. J. Hermann, and S. McNamara (Taylor \& Francis, London, 2005), p. 1029.

[11] B. Thomas and A. M. Squires, Phys. Rev. Lett. 81, 574 (1998).

[12] M. A. Naylor, M. R. Swift, and P. J. King, Phys. Rev. E 68, 012301 (2003).

[13] M. E. Möbius, X. Cheng, P. Eshuis, G. S. Karczmar, S. R. Nagel, and H. M. Jaeger, Phys. Rev. E 72, 011304 (2005). 